\title{
Aproximación crítica a la terapia ocupacional en la era de la información [1]
}

Olga Luz Peñas Felizzola

\section{- Resumen}

Resumen: El objetivo de esta reflexión es revisar las nuevas características del trabajo a nivel global e intentar actualizar o, en caso tal, replantear tanto el concepto manejado en terapia ocupacional, como el quehacer profesional desde esta perspectiva.

Son numerosos los conceptos propuestos sobre lo que es trabajo, no sólo en terapia ocupacional, sino también en múltiples disciplinas. Sin embargo, y a pesar de los esfuerzos transdisciplinarios para mantener un concepto vigente, las nuevas tendencias mundiales obligan a replantear, y casi a rehacer, la construcción tradicional.

En este sentido, comúnmente se ha asumido el trabajo como dimensión en torno a la cual se desarrolla nuestro quehacer; sin embargo, dichos planteamientos entran en clara contradicción con la realidad global, teniendo en cuenta la generalizada tendencia a la reducción y precarización laboral, denominada por algunos como "el fin del trabajo" (1).

Palabras claves: conceptualización de trabajo; historia del trabajo; Revolución Industrial

\section{- Abstract}

Abstract: The goal of this reflection is to revise the new features of the work on a global level and an attempt to actualize or, in such a case, to restate the concept used in occupational therapy, as well as the professional duty from this perspective.

There are many proposed concepts about what work means, not only in occupational therapy, but also in many disciplines. Nevertheless, in spite of inter-disciplines efforts to keep one standing concept, the world's new tendencies impel to restate, and almost to remake, traditional construction.

In this sense, it has been commonly assumed the work as a dimension round about to which to develop our duties; nevertheless, such executions are in clear contradiction with global reality, taking into account the generalized tendency for reduction and laborer precariation, named for many "the end of work".

Key words: work conceptualisation; work history; Industrial Revolution. de Rehabilitación. Docente Maestría en Discapacidad e Inclusión Social, Universidad Nacional de Colombia.

Contacto > > Dirección: carrera 109 A No83-50 Interior 7, apartamento 502, Bogotá, Colombia.

Teléfono: (091) 2274908 Telefax: (091)2867165. E-mail: olgaluzpenas@hotmail.com;

olgaluzpf@starmedia.com 


\section{- Introducción}

Es imperioso para el terapeuta ocupacional conocer y comprender el nuevo significado y formas del trabajo, con miras a reflexionar en torno a las posiciones que vaticinan, incluso, la desaparición de éste. Si ante la tendencia emergente que minimiza la fuerza laboral, releva al trabajo del proceso de producción, reemplaza la producción por el consumo y el trabajo por el ocio, y que replantea el perfil del trabajador, ¿no será también necesario redefinir, desde terapia ocupacional, lo que entendemos y asumimos como trabajo?

Si el trabajo se ha transformado y precarizado $y$, además, nos enfrentamos a la tendencia global del desempleo ¿cuáles serán las expectativas de vida para nuestra profesión que tiene entre sus fines la vinculación de las personas al empleo remunerado?. ¿Qué futuro nos depara si concebimos el trabajo como indicador de éxito de nuestros procedimientos profesionales?

\section{Conceptos y concepciones del trabajo}

\subsection{Perspectiva interdisciplinaria}

Diferentes disciplinas han planteado sus propias consideraciones acerca del trabajo, cada una guardando sus propias particularidades. Sin embargo, a pesar de la variedad y complejidad de los conceptos propuestos, no existe uno de aceptación universal -o por lo menos generalizado-, ya que la construcción y aceptación de cada definición está mediada por diversidad de factores, como son: disciplina, corriente de pensamiento, problemática abordada, incluso por determinantes de tiempo y espacio.

En este sentido, es importante considerar que, "conviene desconfiar de las definiciones metafísicas o muy generales del trabajo, desligadas de la historia, sin referencia a la variedad de sus formas concretas de acuerdo con las sociedades, las culturas, las civilizaciones, sin consideración suficiente de la manera en que el trabajo es vivido y experimentado por quienes lo realizan".

Por ello, la exposición que a continuación se hace de diferentes conceptos de trabajo, responde más al ejercicio de reconocer la variedad de posiciones -contradictorias y afines-, antes que pretender unificar criterios. De todas formas, este recuento permitirá nutrir, con posterioridad, la revisión de la definición manejada por los terapeutas ocupacionales.

Desde la visión ortodoxa, conforme con el dogma católico, y según lo consignado en el antiguo testamento, el trabajo es una maldición impuesta al hombre como castigo por sus pecados: "mediante el sudor de tu rostro comerás el pan, hasta que vuelvas a confundirte con la tierra de que fuiste formado" (므). En este sentido, el pensamiento religioso concibe que el hombre trabajará, casi exclusivamente, con el objeto de sostener la vida de su familia y la suya propia, en respuesta al castigo divino que le fue asignado (ㄴ) .

Perspectivas menos ortodoxas, en este caso la sociológica, distinguen el trabajo especialmente por sus fines y su utilidad, y es considerado "común denominador y 
condición de toda vida humana en sociedad" (2). A partir de este enfoque se ve al trabajo como actividad humana socializante, dada la naturaleza social del ser humano, ocupado esencialmente del trabajo en diversidad de contextos, y niveles de desarrollo y evolución en la estructura económica de las sociedades.

Max Scheler, filósofo, califica el trabajo como "indiferente" y "ciego", el cual, según él, carece de una finalidad universal, siendo imposible conceptualizarlo sin tener en cuenta los grupos sociales y étnicos, los contextos culturales e, incluso, los enfoque y juicios particulares que lo relativizan. Por tanto, sería ambicioso pensar en un concepto suficientemente completo.

La psicología, en su derivación freudiana, plantea el trabajo en términos de "factor de equilibrio psicológico, de estructuración de la personalidad, de satisfacción durable" (2). En este sentido, el trabajo es asumido como "fenómeno decisivo en el ascenso del hombre por encima de la animalidad".

El enfoque económico, en este caso desde la corriente marxista, propone que el trabajo es, esencialmente, la transformación que hace el hombre de la naturaleza, la cual, recíprocamente, reacciona sobre él, modificándolo (ㅁ); es, en complemento, el proceso consciente del ser humano para transformar los materiales de la naturaleza en elementos útiles para su vida. Desde este enfoque, la historia del hombre ha estado acompañada de una continua "lucha de clases", en la cual la sociedad capitalista está dominada por la producción de mercancías que no son otra cosa que trabajo humano materializado. Dentro de esta misma línea de pensamiento se encuentran también los aportes de F. Engels (ㅁ), quien explica el papel decisivo del trabajo en el proceso de hominización Según Engels, el trabajo permitió, entre otras cosas, la producción de instrumentos y la construcción de la sociedad, al tiempo que, incluso, moldeó el tipo físico del hombre. "El trabajo es fuente de toda riqueza (...) lo es, en efecto, a la par que la naturaleza, proveedora de los materiales que él convierte en riqueza. Pero el trabajo es muchísimo más que eso. Es la condición básica y fundamental de toda vida humana. Y lo es en tal grado que, hasta cierto punto, debemos decir que el trabajo ha creado al propio hombre".

En síntesis, aun cuando los conceptos siguen unas directrices propias de la disciplina de origen, es pertinente anotar que, incluso al interior de éstas, existen diferentes corrientes de pensamiento -también denominados enfoques- que siguen ramificando la conceptualización del trabajo.

\subsection{Terapia Ocupacional y Trabajo}

La terapia ocupacional, desde sus mismos orígenes y paralela a su evolución, guarda estrecha relación y fidelidad con el concepto de trabajo y con el abordaje del mismo como derivación de su objeto de estudio disciplinar.

Dos de las primeras definiciones de terapia ocupacional, las cuales incluyen ideas como: "terapia de trabajo activo" (Simon) y "ciencia de organizar el trabajo para inválidos" (Hall) (ㄱ), evidencian la importancia del trabajo como factor denominador de la profesión, aún cuando la perspectiva de su concepción sea de características eminentemente clínicas, coincidentes con el enfoque predominante para la época primeras décadas del siglo XX-. 
Para 1940, Neustadt (ㄱ) propone el trabajo como hábito en la existencia de la persona, que determina no solo su estilo de vida, sino también su satisfacción y estatus social. Esta definición amplía el concepto inicial de trabajo, involucrando ahora componentes personales (motivacionales, comportamentales), así como sociales, superando claramente las restricciones que en estos aspectos presenta el modelo médico.

A partir de 1966, demandas de tipo económico y cultural fortalecieron el papel del terapeuta ocupacional en el sector trabajo, ya que los procesos de industrialización, automatización y urbanización perfilaron un profesional capaz de abordar el bienestar laboral de la población trabajadora, principalmente en lo que se refería a potencializar la productividad en la industria ( $\underline{7})$.

Así, las influencias sociales, económicas y culturales de las últimas cuatro décadas, motivaron cambios en nuestros patrones de abordaje del trabajo, sobre todo en lo que se refiere a la praxis profesional (componente procedimental), ya que a nivel conceptual no se han evidenciado cambios que sugieran un replanteamiento fundamental (ㄱ) .

Conceptos actuales, plantean el trabajo como la actividad con fines de producción o recompensa o aquella que sin ser asalariada contribuye a la subsistencia y producción. Generalmente se encuentra limitado por tiempo y espacio" (요). También como todas "aquellas actividades productivas, remuneradas o no remuneradas, cuya realización por parte de la persona no es opcional, sino que exige de ella cumplimiento de tiempos y normas de ejecución, y que se distingue por su contribución a sí mismo, a otros seres humanos y al progreso social" (ㅁ). Desde esta perspectiva, el trabajo se configura como eje del desempeño ocupacional, hasta el punto de ser asumido como indicador o "meta que expresa el éxito de los procedimientos de terapia ocupacional". Desde esta perspectiva, se asume que "el fin último de los programas de terapia ocupacional es la permanencia, incorporación o reincorporación de las personas al empleo remunerado o al trabajo con fines útiles y de servicio".

Para resumir, son diferentes los enfoques bajo los cuales se pueden enmarcar los conceptos de trabajo en terapia ocupacional, eso sí, desde corrientes más complementarias que contrarias:

a) Desde una perspectiva de la moral, el trabajo figura como proceso que desarrolla la disciplina en el ser humano. Esta corriente imperó principalmente durante los siglos XVIII y XIX en el manejo de las personas con enfermedad mental, quienes eran consideradas "menos humanas" o poseídas por demonios. Este movimiento del tratamiento moral, según diversos autores $(\underline{8}, \underline{10})$, determinó el nacimiento de la terapia ocupacional.

b) El trabajo como fuente que satisface la necesidad innata del hombre de ser productivo (11). Esta corriente adopta la definición de trabajo desde las bases del desempeño y la ocupación, considerando el sentido intrínseco de competencia y maestría que se le atribuye, según los principios filosóficos disciplinares.

c) El trabajo como actividad terapéutica. Conservando la visión clínica dominante (10 $)$, el trabajo se perfila como actividad restauradora de la salud, independientemente de que satisfaga los intereses o expectativas de la persona. El trabajo como remedio natural. 
d) El reduccionismo también influencia en gran medida el concepto de trabajo en terapia ocupacional. Propone el trabajo a partir de la fragmentación del mismo en sus componentes básicos, como son motivación, habilidades, productividad, cultura. De este enfoque se deriva el actual abordaje especializado del trabajo, principalmente en lo concerniente a instrumentos evaluativos; ya que, antes que intentar conservar la integralidad de la persona, atomiza los componentes de ésta y de la ocupación para la aplicación de los diversos procedimientos profesionales.

e) Desde la psicología humanista, la teoría de las necesidades de A. Maslow, determina en gran medida el concepto de trabajo que se maneja en terapia ocupacional, donde cobra especial importancia el principio de satisfacción que, hoy día, le es adjudicado $(\underline{10})$. El trabajo como satisfactor de necesidades.

\section{Formas e historia del trabajo: de la sociedad preindustrial a la segunda revolución industrial}

Desde los orígenes de la civilización, el trabajo ha figurado como eje estructurador de las sociedades alrededor de los sistemas de producción vigentes, aún en los más incipientes. Hoy, por primera vez, estamos presenciando que el trabajo se desliga de la producción debido al acelerado proceso de sustitución del hombre por tecnología.

El concepto de trabajo ha evolucionado incesantemente a través del tiempo. En numerosas sociedades, como en la Grecia clásica y en el feudalismo medieval de occidente, algunas de sus formas (como las tareas manuales), no solo fueron despreciadas, sino que además se delegaron, casi exclusivamente, a las denominadas "clases inferiores". Por el contrario, en la ya disuelta URSS, se promovió por todos los medios el reconocimiento del valor del trabajo manual, y con frecuencia se podía observar la consigna: "el trabajo es una cuestión de honor, de valentía y de heroísmo", con el fin de motivar a las masas para su aceptación y realización (2).

En el transcurrir de la historia reciente, claramente pueden identificarse cuatro periodos en los cuales el trabajo y el proceso de producción han sufrido radicales transformaciones: sociedad preindustrial, Primera, Segunda y Tercera Revolución Industrial. Sin embargo, es de tener presente que los cambios resultantes de estos periodos, más allá de lo estrictamente "industrial", han impactado ampliamente sobre todos los ámbitos de la vida y en todos los puntos cardinales; $y$, antes que ser procesos abruptos, son el resultado de una sumatoria de hechos, inventos y descubrimientos que partieron la historia y nos siguen influenciado hasta hoy (12).

Antes de 1760, previo a la Primera Revolución Industrial, las formas de producción -y, por lo tanto, de trabajo-, se reducían a la actividad artesanal y a la utilización incipiente de la tierra en actividades de agricultura. A nivel general, las formas de producción respondían a patrones de vida eminentemente rural y el sistema se sustentaba en el trabajo humano y la fuerza animal, mediante la utilización de instrumentos de tecnología primitiva. El trabajo, "esencialmente manual, se basaba en el uso de azadas y azadones, de arados tirados por caballos (...) y otros elementos de la misma naturaleza" (1).

Posteriormente, con el desarrollo de la Primera Revolución Industrial (1760 a 1830) [3]], se inició el proceso de sustitución del trabajo humano y fuerza animal, por el 
funcionamiento de las máquinas. En ese momento, aparecieron el carbón como fuente de fuerza mecánica y la máquina de vapor que, rápidamente, colonizaron la industria y se convirtieron en su fuerza primordial. Junto con estos, otros acontecimientos revolucionaron la vida y el trabajo de la época: aumentó la población mundial; las personas del campo se fueron a las ciudades, buscando trabajar en las nacientes industrias; aumentó la especialización de la mano de obra; numerosos instrumentos y máquinas fueron creados para incrementar la producción; se descubrieron nuevas formas de trabajar y utilizar el hierro, entre muchos otros (ㄴ)

La Segunda Revolución Industrial (de 1830 a la Primera Guerra Mundial), trajo consigo la energía generada por el petróleo y la electricidad. La industria, como gran reclutadora de mano de obra, se encontraba en firme expansión, absorbiendo la población obrera.

En síntesis, hasta este momento (mediados del siglo pasado), los procesos de producción experimentaron un progresivo avance en materia de desarrollo tecnológico, con ganancias para los trabajadores en cuanto a garantías sociales y condiciones de bienestar [4]. El industrialismo occidental de tipo clásico que empezó a difundirse y a consolidarse se caracterizó por una organización taylorista, así como por la producción masiva de unos pocos productos, procesos en cadena, contratación fija de personal, pensión por desempleo o jubilación para los empleados, y sueldos con mayor capacidad adquisitiva. En otras palabras, este periodo se desarrolló con relativa estabilidad, sin grandes amenazas o riesgos (13).

Sin embargo, con la Tercera Revolución Industrial, "revolución tecnológica" o "era de la información", surgida después de la Segunda Guerra Mundial y vigente en nuestros días, se implanta y expande el régimen de las "máquinas pensantes" (1). Ahora la tecnología asume las más diversas funciones, anteriormente de dominio humano, remplazando no solamente la fuerza y la habilidad de las manos, sino también complejos procesos mentales.

\section{- 3. El trabajo hoy: ausencia y precarización}

Esta nueva revolución tecnológica caracterizada por numerosos y diversos cambios, nos obliga, como disciplina experta en la ocupación humana y el trabajo, a indagar y reflexionar en torno a los procesos que se están generando y que demandan, con urgencia, asumir una posición crítica sobre el futuro de la terapia ocupacional. Es por ello que a continuación se presentan las características más relevantes del actual momento, desde la perspectiva del trabajo, con miras a determinar los posibles retos para los terapeutas ocupacionales, así como alternativas viables para responder a ellos.

"Hasta hace poco, la informática era cara, poco eficiente, esotérica y, por todo ello, se circunscribía a un número reducido de empresas y de funciones: su elitismo la hacía privativa de los poderosos" (14) [ㄷ]. A partir de la década de los 50, en todo el mundo se experimentó el nacimiento de una nueva revolución industrial, de carácter tecnológico, que, desde sus inicios, rompió los esquemas tradicionales de producción, replanteó el trabajo -desligándolo del proceso de producción-, y popularizó la tecnología. Con los avances tecnológicos de este nuevo periodo, se experimenta el cierre de numerosos puestos de trabajo, lo cual ha conllevado a que, día a día, sea 
menor la oferta de empleos, y mayor el número de personas cesantes. Este fenómeno ha sido planteado por algunos como el fin del trabajo.

Ahora, la información se consolida como nuevo centro de interés del sistema: es la nueva mercancía. Transmitida globalmente en tiempo real, su manejo, acceso y acumulación se configuran como poder estratégico.

Visto desde otro punto, este nuevo momento histórico ha traído consigo la diversificación de la producción de bienes y servicios, adaptados a las nuevas y cambiantes demandas del mercado; así como una acelerada proliferación de innovaciones científico-tecnológicas y la exigencia de un nuevo trabajador: polivalente, autónomo y participativo ( $\underline{15})$. Nos enfrentamos, de manera irreversible, a la era de la información y la tecnología.

Con el objeto de construir un panorama general del trabajo en la era tecnológica, a continuación se exponen los aspectos de mayor relevancia que lo caracterizan para, posteriormente, analizarlos a la luz de la terapia ocupacional.

\subsection{El sistema de producción}

Uno de los cambios radicales del sistema consiste en el paso de una economía basada en los materiales, en la energía y en el trabajo, a una basada en la información y en la comunicación. Esto reorienta los procesos de formación y ubicación laboral y, desde el quehacer del terapeuta ocupacional, se requiere revisar hacia dónde estamos enfocando la orientación, capacitación y ubicación laboral, con el fin de verificar que realmente estén apuntando a las nuevas exigencias, es decir, una economía que privilegia la informática y las comunicaciones.

El actual sistema se caracteriza por la continua reinvención y eliminación de ocupaciones y tareas. Debido a la automatización, se observa una reorganización de los procesos de producción existentes, así como el nacimiento de nuevas ocupaciones que obedecen a la aplicación de los avances científico-tecnológicos en la industria. Dichos avances han impactado, además, flexibilizando y desespecializando el trabajo, agilizando el proceso de producción y multiplicando las tareas del puesto, mientras ejercen el control que antes estaba en manos del trabajador, programando instrucciones y verificando el cumplimiento de las mismas. A medida que avanzan la ciencia y la tecnología se requiere también que las personas y los procesos se adapten a las nuevas condiciones y exigencias ( $\underline{15})$.

Para el terapeuta ocupacional, esta situación plantea el reto de promover la flexibilidad de la persona para un desempeño eficiente; ello es una ruptura a la visión tradicional reduccionista que encasilla al trabajador en un "puesto de trabajo", cuando en la realidad son difusos los límites entre unos y otros. La respuesta de los terapeutas sería de doble vía: por un lado, dirigido a replantear los procedimientos profesionales tendientes a diagnosticar, de manera puntual, los puestos de trabajo y, por otro, para el proceso de orientación, capacitación y ubicación laboral, el cual deberá ser visto desde una óptica más amplia que favorezca la movilidad ocupacional del usuario en las áreas ocupacionales que privilegia el mercado.

En resumen, "las nuevas exigencias en el desempeño competente de los trabajadores han delatado la obsolescencia de los métodos científicos de análisis de puestos (...) la 
alta especificación de tales análisis riñe con la flexibilidad requerida en el desempeño

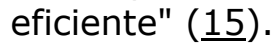

\subsection{El empleo $y$ el desempleo}

Desde la gran depresión de los años 30, el desempleo nunca había alcanzado niveles tan elevados como los de hoy; fenómeno que tiende a incrementarse como consecuencia de la revolución tecnológica que, rápidamente, ha remplazado la mano de obra, por las tecnologías basadas en la información, sin discriminar sector económico (1). Este fenómeno, también llamado "desempleo tecnológico", deriva de la implantación de nuevas tecnologías que remplazan la mano de obra y eliminan puestos de trabajo pues, aún cuando en la actualidad se están creando oportunidades de empleo, no son suficientes para absorber la demanda de desempleados, cada vez más creciente.

"El rápido camino a la automatización conduce vertiginosamente a la economía global a un futuro industrial sin trabajadores" (1 $)$. Tal es el desempleo en el mundo, que se han debido revisar y ajustar sus indicadores y replantear el concepto de persona ocupada o trabajando, con el fin de disimular las alarmantes cifras de cesantes, subempleados e informales. La inseguridad en el empleo (alta rotación y escasez) es otro denominador del mercado laboral, caracterizado por trabajos temporales y periodos de receso cada vez mayores, entre uno y otro.

El pleno empleo es ahora una utopía superada, no por que se hubiera logrado, sino por ser inmensamente distante de la realidad.

Teniendo en cuenta dicho fenómeno, urge reflexionar desde terapia ocupacional sobre cuál será el papel a jugar en un mundo sin trabajo, partiendo de la relevancia que éste tiene como eje del desempeño ocupacional, como indicador de éxito de nuestros procedimientos profesionales y como fin último de los programas de intervención. Ante esta situación, conviene revisar la pertinencia del terapeuta ocupacional en los procesos de inserción laboral, ya que, si el objetivo de dicho proceso es ubicar y mantener a la persona en un empleo, serían resultados frustrantes los que se alcanzarían ante una inclusión inacabada, precisamente por la ausencia de una opción de trabajo.

En respuesta a lo anterior, diversos autores (1) le apuestan al denominado "tercer sector", o economía no basada en el mercado (o economía social), como respuesta viable a las necesidades personales y sociales para explorar nuevos roles y responsabilidades, tendientes a encontrar un nuevo valor de la persona ( $\underline{1})$. Este aspecto apunta a convertirse en una opción viable para insertarse en el nuevo mercado que privilegia los servicios, al tiempo que responde a la búsqueda de un rol social, ya no derivado del trabajo, sino con la revaluación del tiempo libre.

\subsection{Los trabajadores}

El anterior patrón de producción, de predominante corte fordista, ha sido desplazado por la tendencia a la reducción y sustitución del trabajo humano. La automatización y la robotización aplicadas a la producción, trajeron consigo la disminución de la mano de obra requerida, ya que esta tecnología permite incrementar la productividad y las utilidades con menos recurso humano. La persona, como fuerza del sistema de producción, tiende a perder su vigencia. 
La velocidad con la cual avanzan la ciencia y la tecnología de hoy, requiere que el trabajador moderno responda con la misma agilidad. Por ello, día a día, el mercado de trabajo exige personal cada vez más capacitado, flexible y polivalente, de manera que pueda asumir y responder a varias situaciones de diversa complejidad, sin circunscribirse a un solo puesto de trabajo.

Autores como Kielhofner y Johnson, y Monfette $(\underline{10})$ identifican para terapia ocupacional las posibles situaciones a enfrentar a partir de las tendencias del trabajo a nivel global. Como consecuencia de los avances tecnológicos, explican, cada vez resultará más difícil que la persona encuentre satisfacción en los roles productivos tradicionales, lo cual podría condicionar la creatividad, habilidades y destrezas en el trabajo. Desde esta perspectiva, los avances tecnológicos y la misma "informatización" de la sociedad traerán consigo, no solamente el problema del desempleo, sino que, día a día, será más difícil acceder y conservar el trabajo, ante el cierre de puestos.

De otro lado, el nuevo trabajador deberá asumir tantas y tan variadas funciones y responsabilidades, que, difícilmente, alcanzará maestría o dominio en algún área. Ante esto, los terapeutas ocupacionales deberemos responder a las nacientes necesidades locales y globales que demandan identificar nuevas fuentes de productividad, satisfacción, realización personal y estatus social, antes propias del trabajo, hacia otros roles y áreas de la ocupación humana.

\subsection{La salud y bienestar}

Todo este proceso de cambio ha traído consigo, obviamente, implicaciones sobre el bienestar de la población trabajadora. Estudios realizados en la Universidad de Utah (USA) identificaron una fuerte asociación entre la pérdida del empleo y el incremento de las enfermedades cardiovasculares (infartos y ECV): el "incremento en un punto porcentual en el desempleo, desencadena un 5,6\% de incremento en las muertes como consecuencia de ataques al corazón; y un 3,1\% de incremento en las muertes como consecuencia de apoplejías. Además, las personas desempleadas están más predispuestas al estrés y la depresión, a consumir más alcohol y cigarrillo, y a comer con dietas menos saludables" (1).

De igual forma, una investigación desarrollada en la Universidad de Winsconsin identificó que los trabajadores supervisados por medios electrónicos son más propensos, aproximadamente en un $15 \%$, a padecer depresiones, tensión nerviosa y ansiedad extrema, los cuales pueden derivar en hipertensión, úlceras e infartos. Estudios del National Institute of Occupational Safety and Health -NIOSH, han demostrado la asociación entre el uso del computador y preocupantes problemas de estrés. Otros encontraron nexos entre el desempleo tecnológico y crecientes niveles de depresión y "pesimismo psicótico" ( $\underline{1})$.

Anteriormente era la fatiga física la que aquejaba al trabajador. Hoy, es la fatiga mental producto del "ritmo del nanosegundo" que programa a las nuevas tecnologías. Una analogía que representa la velocidad con la que se mueve el mundo moderno de la informática es presentada por G. Simons (1 $)$ : "imaginemos (...) dos computadores hablando entre sí en un determinado periodo de tiempo. En un momento dado, un ser humano les pregunta sobre lo que están hablando, y en el tiempo que éste tarda en efectuar la pregunta, ambos computadores habrán intercambiado más palabras que las que pueden haberse intercambiado los seres humanos desde que el primer Homo sapiens hizo su aparición sobre la tierra, hace 2 ó 3 millones de años". 


\subsection{El tiempo libre}

"Después de siglos de definir el valor del ser humano en términos estrictamente productivos, la completa sustitución del trabajo humano por máquinas deja a los trabajadores sin autodefinición válida o función social" (1 1 ). Frente a la posibilidad del desempleo o una jornada laboral recortada, la utilización del tiempo libre se perfila como tema de gran valor en el entorno social y político. Varios autores explican que la necesidad de responder a la carencia de empleo masivo formal constituirá el "elemento de presión social más importante" para este nuevo siglo (1). Ante esta situación, inevitablemente se deberá responder a las consecuencias duales de la nueva dinámica laboral: una sociedad "desempleada", o una sociedad "liberada" del trabajo, lo cual dependerá de las medidas que se tomen para enfrentar tal fenómeno.

Hace casi cinco décadas ya se pronosticaba la necesidad de identificar nuevas fuentes de posicionamiento y significado social alternas al trabajo, ya que, hasta el momento, éste había sido indicador del valor social (4). "Si el trabajo puede llegar a ser más difícil de conseguir, necesitaremos revaluar nuestras actitudes hacia el trabajo y descubrir otros modos de expresar nuestro sentido de pertenencia y contribución a nuestra sociedad" (4). Tales cambios implicarían redefinir el trabajo y replantear, desde el sistema educativo, hasta la utilización del tiempo libre y la formación para el trabajo, cuyo resultado sería una modificación radical de la asignación de valores, y una forma de vida incompatible con los preceptos tradicionales. Se avecina un obligatorio incremento en el tiempo libre, desempleo y ocio, cuando, durante siglos, el valor de la persona estuvo dado en términos de producción. En este mismo sentido, nos urge, en términos de Rifkin, identificar nuevas formas para definir el valor del ser humano y sus relaciones, pues el trabajo, como categoría de autodefinición y valoración de la persona, apunta a perder su vigencia.

"La automatización amenaza con hacer posible la inversión de la relación entre el tiempo de ocio y el de trabajo: esto es, hacer que el tiempo empleado en el trabajo se convierta en marginal, mientras que el tiempo empleado en el ocio se haga fundamental" (1 $\underline{1})$. Herbert Marcuse, filósofo y psicólogo alemán, anticipó 50 años atrás la realidad que hoy se discute y que, desde nuestra perspectiva disciplinar, requiere un análisis detallado dada la importancia actual y el impacto que, a futuro, puede tener sobre la reformulación de los derroteros profesionales: la inversión de la relación trabajo - ocio (16).

Contradictoriamente, "en la actualidad se teme a la sustitución del trabajo humano por las máquinas, porque todavía no se sabe cómo solucionar el problema del desempleo por la reducción del tiempo de trabajo" (16). Para terapia ocupacional, el esparcimiento $u$ ocio se asume como las actividades realizadas en el tiempo excedente de trabajo (9 $)$, es decir, casi como oposición a éste. Pero, con la tendencia actual, será el ocio el que reemplace la relevancia y el valor social que le ha correspondido al trabajo. Ante esto, es necesario plantear el interrogante de hacia dónde encausar nuestro actuar: ¿hacia una intervención conducente al trabajo o hacia el ocio y el tiempo libre?. Este planteamiento se fortalece también con los indicadores de desempleo actuales, pues, ante la sobreoferta de mano de obra y la escasez y cierre de puestos de trabajo, ¿cuál sería el objetivo de realizar un proceso de inclusión laboral, por demás costoso (en tiempo y recursos) si no logrará su cometido central de mantener -o, por lo menos, ublicar- a la persona en un trabajo determinado? 
Si el valor que se le asigna socialmente al trabajo responde al criterio de identificar satisfactores para las necesidades humanas fundamentales: de participación, ocio, protección, subsistencia, afecto o creación, sería más sensato cambiar el satisfactor (17), en este caso el trabajo, ante la dificultad de acceder a él; teniendo en cuenta que las necesidades fundamentales a las cuales respondía siguen vigentes -por su misma naturaleza son constantes-, pero la validez de éste, económicamente ha caducado y culturalmente se está redefiniendo. Queda por resaltar la importancia que, desde terapia ocupacional, tiene la ocupación $y$, por lo mismo, la habilidad y capacidad disciplinar para identificar, desde la argumentación y la praxis, nuevas opciones de desempeño ocupacional con potencial de valoración y definición social de la persona.

\section{Consideraciones finales}

Sin pretensión de convertirlas en conclusiones, sino como punto de partida para reflexiones futuras, a continuación se proponen una serie de ideas claves, resultantes de este ejercicio reflexivo:

- Vale la pena retomar la significación y etimología de "ocupación", dado que hace parte integral del nombre de nuestra disciplina. En primer lugar, "ocupación" no es, necesariamente, sinónimo de "trabajo", entendido éste como "ocupación retribuida". Ocupación, en una de sus múltiples acepciones, y la más pertinente para este efecto, es, simplemente "actividad, entretenimiento" (18). La Terapia ocupacional, entonces, no estaría dirigida necesariamente a la inserción en el mercado laboral, sino a la búsqueda de nuevas formas de realización plena de los seres humanos en el ejercicio de sus actividades, productivas, pero también lúdicas, o simplemente ociosas; orientadas al ejercicio pleno de la humanidad.

- Conviene reflexionar en torno a nuevas formas para definir el valor del ser humano y El trabajo, como realidad y como concepto, es constantemente cambiante y relativo. Por ello, desde terapia ocupacional, es imprescindible mantener una dinámica de reflexión y crítica, desde una perspectiva transdisciplinaria, que asegure la coherencia entre la realidad y nuestro quehacer profesional.

- sus relaciones, pues el trabajo, como categoría de autodefinición y valoración de la persona, apunta a perder su vigencia.

- Ante las nuevas tendencias del mercado laboral que apuntan a un futuro sin trabajo, y que han minimizado al trabajo como indicador del valor social de la persona o como referente de socialización y productividad, surge el dilema sobre la finalidad de la disciplina ocupacional: ¿terapia ocupacional para el trabajo, o terapia ocupacional para el ocio?

- Urge reflexionar desde terapia ocupacional sobre el papel a jugar en un mundo sin trabajo, partiendo de la relevancia que éste tiene como eje del desempeño ocupacional, como indicador de éxito de nuestros procedimientos profesionales y como finalidad de los programas de intervención. Conviene, también, revisar la pertinencia de los procesos de inserción laboral, ya que, una inclusión no alcanzada se traduce en un resultado frustrante, muy diciente de la pertinencia de nuestra intervención.

- La economía no basada en el mercado (o tercer sector), así como la revaluación del tiempo libre, son opciones válidas en la búsqueda de un nuevo rol 
ocupacional, diferente al trabajo, que responda a las necesidades de productividad, satisfacción, realización personal y estatus social.

"Interpretar erróneamente el presente, tan solo como una continuación de la industrialización, en lugar del nacimiento de una nueva era tecnológica, asegura una incapacidad de anticiparse a un sistema completamente distinto de las relaciones humanas".

J. Rifkin, 106.

\section{- Bibliografía}

1 Rifkin, J. (1996). El Fin del Trabajo. Barcelona: Ediciones Paidós.

2 Friedmann, G. y Naville, P. (1997). Tratado de Sociología del Trabajo, vol. 1 y 2. México: Fondo de Cultura Económica.

3 La Biblia. (1995). Bogotá: Paulinas.

4 Brown, J.A. (1998). La Psicología Social en la Industria ( $3^{\circ}$ ed.). México: Fondo de Cultura Económica, colección Breviarios.

5 Marx, C. (1985). El Capital. México: Fondo de Cultura Económica.

6 Engels, F. El Papel del Trabajo en la Transformación del Mono en Hombre.

7 Marshall, E. (1985). Looking Back. Work Evaluation as a Theme. En: American Journal of Occupational Therapy, vol.39, Núm. 5, mayo, págs. 295-300.

8 Hopkins, H. Y Smith, H. (1998).Terapia Ocupacional. Madrid: Editorial Médica Panamericana.

9 Trujillo A. (2002). La Terapia Ocupacional: conocimiento y práctica en Colombia. Bogotá: Universidad Nacional de Colombia.

Harvey-Krefting, L. (1985). The Concept of Work in Occupational Therapy: a

10 historical review. En: American Journal of Occupational Therapy, vol.39, Núm. 5, mayo, págs. 301-307.

11 Meyer, A. (1977). The Philosophy of Occupational Therapy. En: American Journal of Occupational Therapy, Núm.31, págs. 639-642.

12 Ashton, T. (1996). La Revolución Industrial (2a ed.). México: Fondo de Cultura Económica, colección Breviarios.

13 Bonastre, R; Palau, X, y Subirats, J. (1996). Manual de Seguridad y Salud en el Trabajo: nuevos conceptos. Barcelona: Editorial Ariel, colección Economía.

14 Nora, S y Minc, A. (1992). La Informatización de la Sociedad. México: Fondo de Cultura Económica, colección Popular.

Organización Internacional del Trabajo (OIT) - CINTERFOR. (2003). Conceptos

15 Básicos de Competencias Laborales. Documento web: www.cinterfor.org.uy/public/spanish/region/ampro/cinterfor/temas/complab/xxx x/esp/i/index/.htm

Sue, R. (1987). El Ocio. México: Fondo de Cultura Económica, colección 16 Breviarios. 
17 Max -Neef, M. (1984). La Economía Descalza: señales desde el mundo invisible. Real Academia Española. (2003). Diccionario de la Lengua Española. Madrid: 18 Espasa.

\section{- Notas}

[1] Este trabajo fue presentado en el XIII Congreso Nacional de Terapia Ocupacional; Bogotá, mayo de 2003. Una versión en inglés está siendo revisada para publicación en el Boletín de la World Federation of Occupational Therapy.

[3] Aunque esta es la delimitación temporal estricta que se establece para la Primera Revolución Industrial, obedece más a criterios históricos que al real desarrollo de la misma. Esta Revolución Industrial, nacida en Inglaterra, surge como consecuencia de un amplio proceso de cambio y reanimación a nivel político, social, intelectual y económico, el cual tuvo sus inicios mucho antes de 1760 y transcurrió hasta bastante después de 1830, el cual, debido a su magnitud y relevancia, se irradió rápidamente hacia todas las latitudes.

[4] Con posterioridad a la Primera Revolución Industrial, las luchas sindicales y gremiales en todo el mundo, en unas regiones más que en otras, paulatinamente habían ido ganando terreno en materia de seguridad social, condiciones de trabajo y salud e, incluso, participación política. Esto repercutió en un notable cambio para los trabajadores, quienes fueron reconocidos como fuerza viva dentro del sistema de producción. Sin embargo, esta situación cambió con la revolución tecnológica de mediados del siglo $\mathrm{XX}$, en detrimento de la clase trabajadora -ahora en vías de extinción-.

[5] Es importante tener en cuenta que estas apreciaciones se dieron en 1978, época en la cual se elaboró este estudio a petición del gobierno francés, con el fin de hacer las proyecciones del impacto de la informática en el país. 